\title{
Collaborative Visualization for Large-Scale Accelerator Electromagnetic Modeling
}

DOE Award Number: : DE-FG02-08ER85143

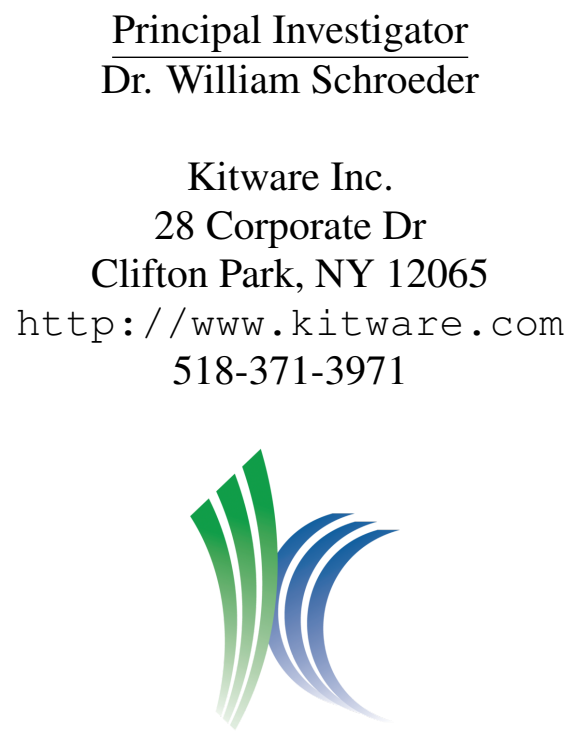

Report Date: September 30, 2011

Period Covered: August 2009 - August 2011 


\section{Executive Summary}

This report contains the comprehensive summary of the work performed on the SBIR Phase II, Collaborative Visualization for Large-Scale Accelerator Electromagnetic Modeling at Kitware Inc. in collaboration with Stanford Linear Accelerator Center (SLAC).

The goal of the work was to develop collaborative visualization tools for large-scale data as illustrated in the figure below. The solutions we proposed address the typical problems faced by geographicallyand organizationally-separated research and engineering teams, who produce large data (either through simulation or experimental measurement) and wish to work together to analyze and understand their data. Because the data is large, we expect that it cannot be easily transported to each team member's work site, and that the visualization server must reside near the data. Further, we also expect that each work site has heterogeneous resources: some with large computing clients, tiled (or large) displays and high bandwidth; others sites as simple as a team member on a laptop computer.

Our solution is based on the open-source, widely used ParaView large-data visualization application. We extended this tool to support multiple collaborative clients who may locally visualize data, and then periodically rejoin and synchronize with the group to discuss their findings. Options for managing session control, adding annotation, and defining the visualization pipeline, among others, were incorporated. We also developed and deployed a Web visualization framework based on ParaView that enables the Web browser to act as a participating client in a collaborative session. The ParaView Web Visualization framework leverages various Web technologies including WebGL, JavaScript, Java and Flash to enable interactive 3D visualization over the web using ParaView as the visualization server.

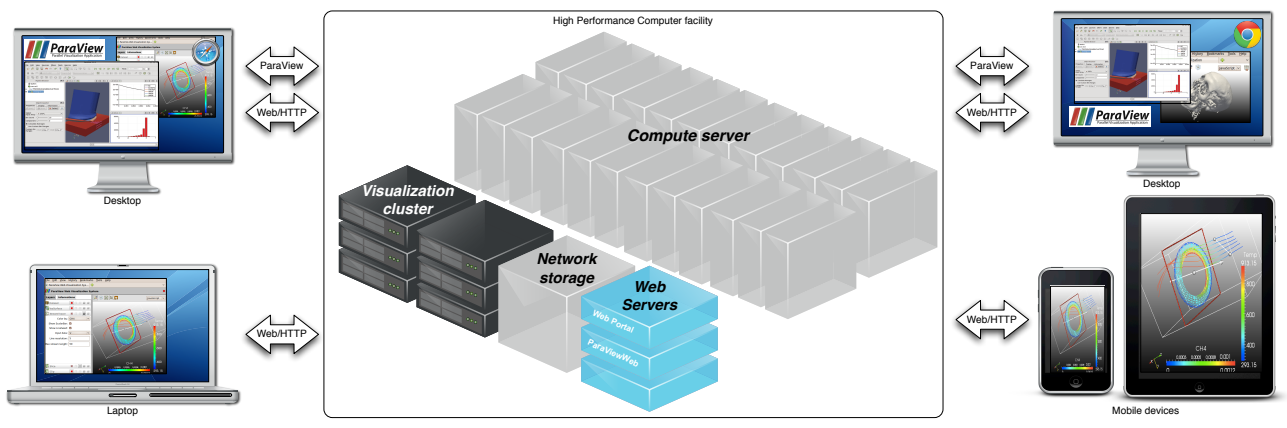

Figure 1: A typical setup for collaborative visualization using ParaView with heterogeneous devices.

We steered the development of this technology by teaming with the SLAC National Accelerator Laboratory. SLAC has a computationally-intensive problem important to the nations scientific progress as described shortly. Further, SLAC researchers routinely generate massive amounts of data, and frequently collaborate with other researchers located around the world. Thus SLAC is an ideal teammate through which to develop, test and deploy this technology. The nature of the datasets generated by simulations performed at SLAC presented unique visualization challenges especially when dealing with higher-order elements that were addressed during this Phase II.

During this Phase II, we have developed a strong platform for collaborative visualization based on ParaView. We have developed and deployed a ParaView Web Visualization framework that can be used for effective collaboration over the Web. Collaborating and visualizing over the Web presents the community with unique opportunities for sharing and accessing visualization and HPC resources that hitherto with either inaccessible or difficult to use. The technology we developed in here will alleviate both these issues as it becomes widely deployed and adopted. 


\section{Contents}

1 Introduction 3

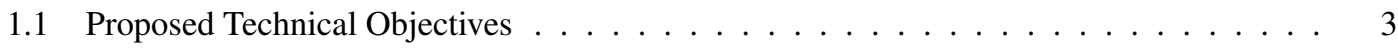

1.2 Work Plan . . . . . . . . . . . . . . . . . . . . 3

1.3 Differences between accomplishments and original proposal $\ldots \ldots \ldots \ldots \ldots$

2 Technical Achievements 4

2.1 ParaViewWeb: Web Visualization with ParaView . . . . . . . . . . . . . . . . . 4

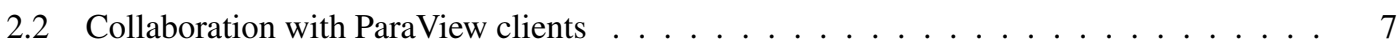

2.3 Collaboration over web (e.g. with web clients) . . . . . . . . . . . . . . . 10

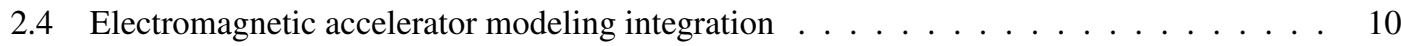

2.5 Deployment . . . . . . . . . . . . . . . . . . . . . 12

3 Publications $\quad 13$

4 Internet Sites 13 


\section{Introduction}

The goal of the effort was to develop collaborative visualization tools for large-scale data as illustrated in the figure below. The solutions we developed addresses the typical problems faced by geographicallyand organizationally-separated research and engineering teams, who produce large data (either through simulation or experimental measurement) and wish to work together to analyze and understand their data. Because the data is large, data cannot be easily transported to each team members work site, and that the visualization server must reside near the data. Further, each work site has heterogeneous resources: some with large computing clients, tiled (or large) displays and high bandwidth; others sites as simple as a team member on a laptop computer.

The solution developed here is based on the open-source, widely used ParaView large-data visualization application. We extended this tool to support multiple collaborative clients who may locally visualize data, and then periodically rejoin and synchronize with the group to discuss their findings. Of course, options for managing session control, adding annotation, and defining the visualization pipeline, among others, will be available.

In this section we reprise our original objectives proposed in the Phase II proposal and provide a comparison of the goals attained and the original proposal. We discuss the technical activities in Section 2.

\subsection{Proposed Technical Objectives}

The technical objectives proposed in our Phase II proposal were as follows:

1. Develop collaboration support in ParaView

2. Specialize the platform for electromagnetic accelerator modeling.

3. Deploy the technology.

The first objective involves extending the system architecture defined in the Phase I effort, elaborating on the current demonstration technology, and implementing the collaboration framework in a robust manner, including establishing tests and a deployment process. To realize the second objective, the team will build adapters to integrate SLACs analysis codes into the collaborative visualization framework. Further, SLAC will provide data and real-world evaluation of the system to ensure that the technology serves the needs of the research community. Finally, we will work with SLAC and Argonne National Lab to deploy the technology to a wider audience.

The overarching goal of this effort is to develop a robust, complete solution for collaborative visualization. The Phase I results have shown that the technology is feasible, hence we are confident that at the end of the Phase II effort we will have a viable solution for large-scale, collaborative visualization. The following section describes how we will achieve this result.

\subsection{Work Plan}

With these objectives in mind, we proposed the following work plan in our original Phase II proposal:

1. Collaboration with ParaView clients

2. Electromagnetic Accelerator Modeling (SLAC) integration

3. Integration with instant messaging applications

4. Collaboration over the web (e.g. with web clients)

5. Deployment to partnering organizations

6. Produce a final report and documentation 


\subsection{Differences between accomplishments and original proposal}

Our milestones/accomplishments are well aligned with out original proposal. The only item in our original workplan that we had to abandon was intergration with instant messaging applications. As we had reported in our SBIR Phase II Annual Progress Report dated May 13, 2010, we had to drop support for using instant messaging clients such as Skype ${ }^{\mathrm{TM}}$ or Yahoo! Messenger ${ }^{\mathrm{TM}}$. Not long after our proposal, both Skype and Yahoo deprecated their plugin APIs and are either totally dropping support for it or undergoing a major overhaul. Integration with IM clients was only a minor part of our original proposal and does not affect the usability or effectiveness of the developed framework if any way. Hence we decided to drop this deliverable. Instead focus of developing a stronger Web visualization framework that when used in conjunction with collaboration support, bring collaborative visualization to tablets and net-books with ease.

\section{Technical Achievements}

In this section we discuss the major milestones in our Phase II development effort. Most of the code developed is either already released and integrated into the public repositories or is on its way to becoming release soon, unless noted otherwise.

\subsection{ParaViewWeb: Web Visualization with ParaView}

In recent years organizations such as DOE and NSF have been advocating infrastructures that include high performance computing resources distributed across the country (e.g. TeraGrid). While this has made it possible to run large simulations, it has resulted in increasingly large amounts of data. To address the problem of large data, visualization technologies have been developed. Visualization involves converting data and information into images that the human visual system may then interpret. Using visualization tools, researchers can understand immense and complex data in ways that would be impossible by looking at the raw data.

Despite advances in visualization technology, several challenges remain if emerging distributed computational resources are to be effectively utilized. First, due to the size and the resulting difficulty in transporting these massive data, there is a growing trend to co-locate the visualization infrastructure with the data-producing supercomputers. Second, project teams are typically geographically and organizationally dispersed. This means that the visualization system must be distributed, and that methods for collaboration amongst the team members must be supported.

TeraGrid-like, high performance computing infrastructures typically deploy a plethora of visualization tools for support the exploration of the results from the simulation runs. However almost all of these tools, including ParaView, are desktop applications that require the users to have use an installation of the tool on their desktop and use some server-side component that runs on the visualization clusters for parallel processing of the data. The resulting lack of collaboration is clear, but also the complexity involved in getting started with the visualization can be tedious. Users have to have the correct version of the client deployed on their desktop then deal with any firewall related issues for communicating with visualization cluster and so on. As an alternative to this workflow, we are advocating a web-based visualization solution.

Web-based technologies are among the most popular field of innovation in the recent years. What makes Web most attractive is the fact that it becoming pervasive across almost all computing applications ranging from communicating via email to paying household bills to streaming movies to mobiles phones. This means that more and more devices are becoming available that are web-enabled. In a ideal world, a scientist can just pop out his mobile device, $\log$ on to TeraGrid (or similar) portal, click on a couple of links to locate the datasets dumped out by her simulation run and then another click to visualize results. Thus very quickly the scientist can engage a dataset using a simple UI to tweak parameters, interact and 


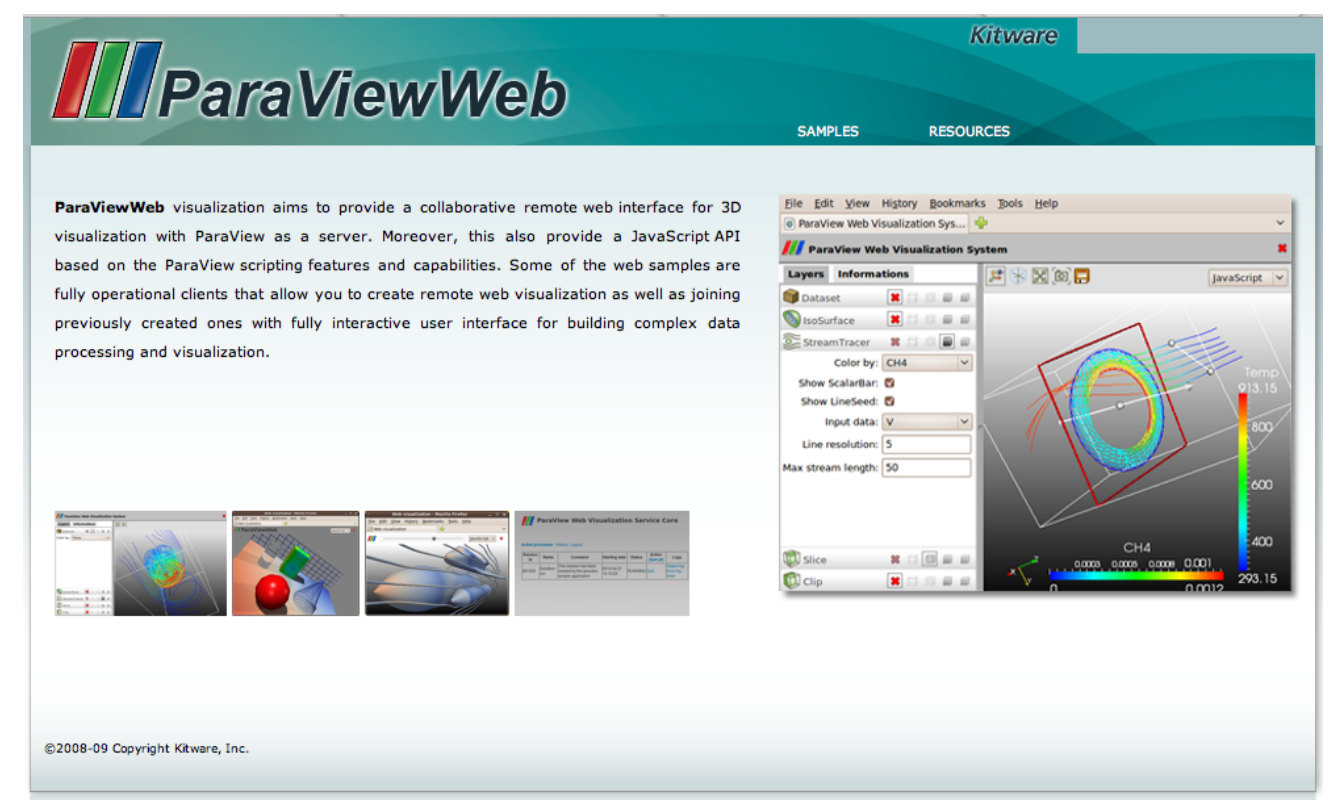

Figure 2: Interactive 3D visualization through the Web browser using ParaViewWeb

explore it. Further, the scientist can quickly communicate with other scientists to join in to explore a dataset, doing so without leaving the omnipresent web-browser environment.

An essential component for collaborative data analysis over the Web is an Web visualization framework that enables interactive 3D visualization through a web browser. For this milestone, we developed such a system, ParaViewWeb.

ParaViewWeb uses ParaView as the parallel data-processing and rendering engine to generate visualizations on the HPC resource while rapidly delivering images to the client. On WebGL enabled browsers, while rendering modest sized geometries, ParaViewWeb can be made to delivery geometries to the web browser and rendered locally. ParaViewWeb makes it easier to develop customized applications for the web that cater to a wide variety of scientists and domain experts. People without the familiarity with complex visualization tools like ParaView and VisIt can analyze their datasets without leaving the familiar and omnipresent environment of the web browser.

The main features of ParaViewWeb are:

- Based on ParaView parallel visualization framework:

- This exposes all of ParaView's visualization capabilities for web-visualization including volume rendering, parallel data processing, distributed rendering and not merely a convenient subset.

- ParaView does all the data processing and rendering, hence we leverage all the benefits of parallel processing.

- This facilitates collaboration with ParaView desktop applications as described in Section 2.3.

- HTTP/HTTPS Communication

- Makes it easier to deal with firewall issues. We don't require any extra ports to be exposed to the outside world, except the HTTP port on which a standard Web server is listening. All interactive visualization communication is routed over HTTP, easing site administrators' concerns about security. 


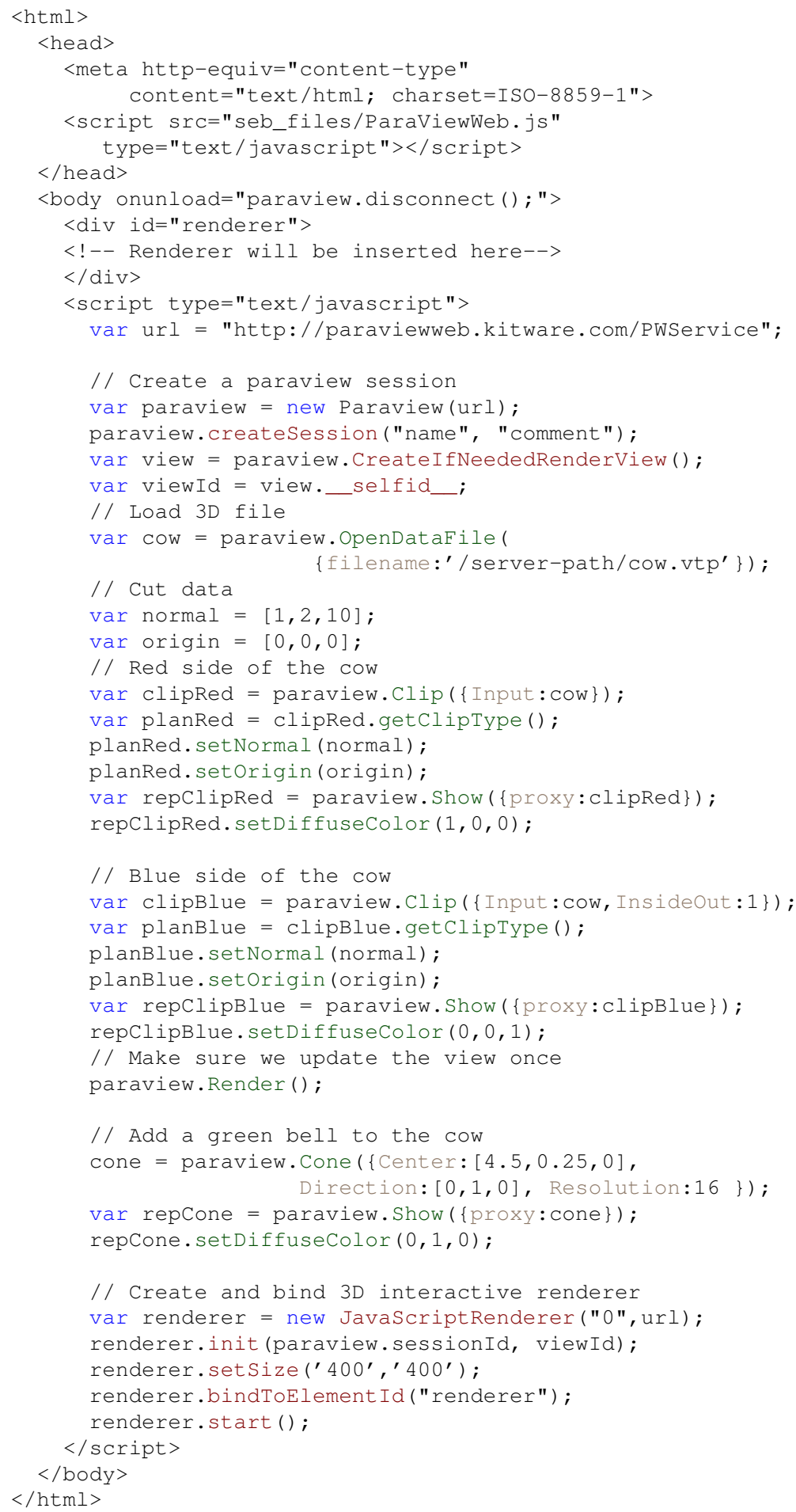

Figure 3: A sample HTML webpage demonstrating the ParaViewWeb JavaScript API to create a simple visualization pipeline for interactive visualization in a Web browser 

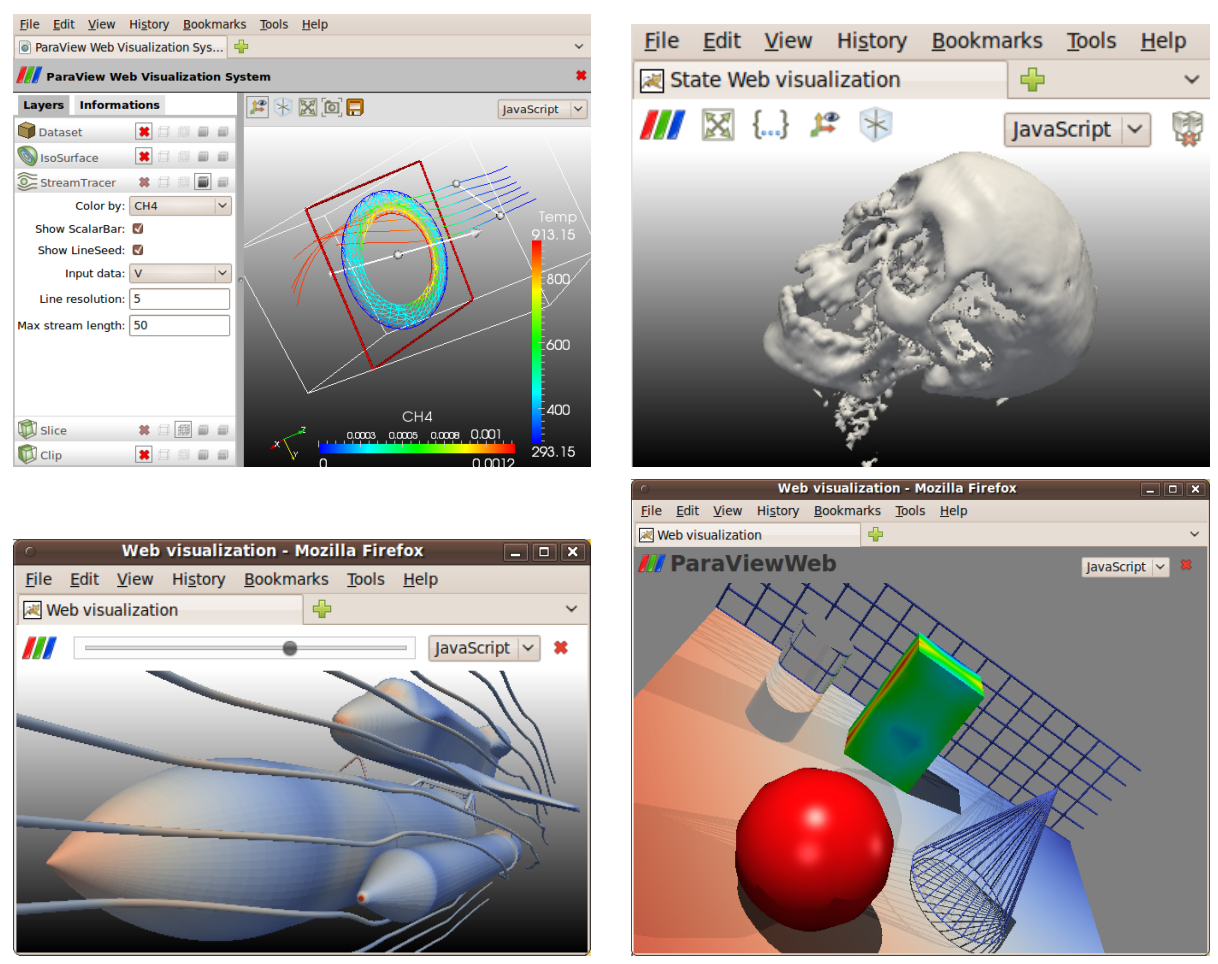

Figure 4: A selection of sample web-applications developed using ParaViewWeb to demonstrate the capabilities of ParaViewWeb.

- Reusable Components

- We have developed a collection of components, rather than a web infrastructure, that can be used within any web portal.

- This allows developers to create custom web-visualization clients suitable for the workflows for the targeted researchers.

- Extensive use of Web standards

- Whenever possible we have used established web standard like WebGL, JSON-RPC, Java Messaging Service (JMS).

- Multiple interactive visualization components

- We have implemented different versions of the interactive 3D visualization component using Java, JavaScript, Flash and WebGL to make it easier to integrate into existing web applications as well develop new ones with ease.

Do demonstrate the use of the ParaViewWeb framework, we developed several custom applications using different Web UI toolkits. Some of these applications are shown in Figure ??. Figure 5 shows a page from the ParaViewWeb administration web application that can be used to monitor active visualization sessions as well as past sessions.

\subsection{Collaboration with ParaView clients}

ParaView already has extensive visualization capabilities (based on VTK) such as isosurfaces, cut-planes, and streamlines. It also has a client-server architecture that is a natural fit for multiple collaboration 


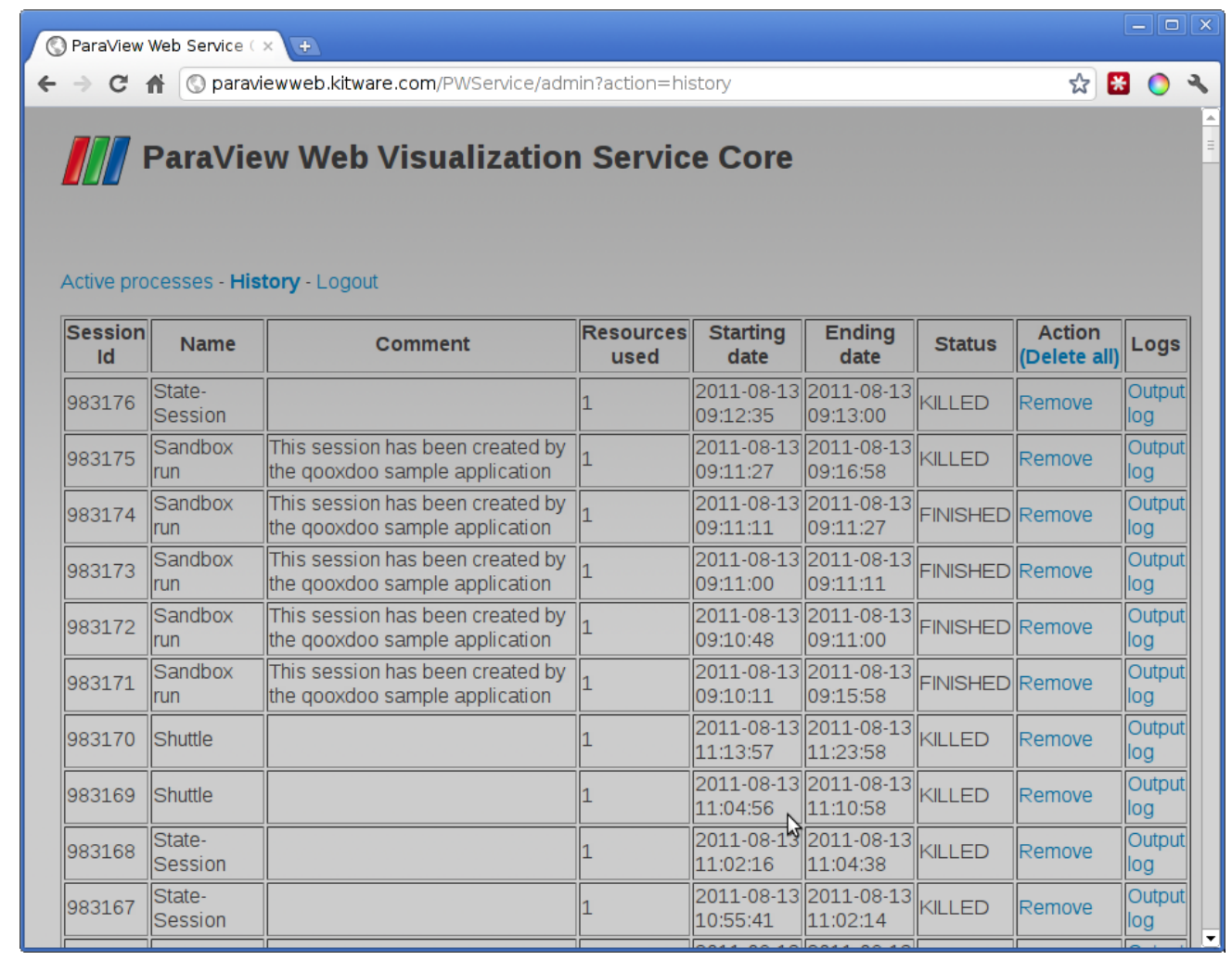

Figure 5: ParaViewWeb administration web-page used to monitor active sessions as well as track past sessions.

clients. However when a server is working with a single client, there are several assumptions that can be made that break down and cause race conditions once we start dealing with multiple clients e.g. the client A can request a render for while client B is performing a render, or, client A deletes a view while client $\mathrm{B}$ is trying to render that view. Such race conditions can cause havoc at runtime and lead to segfaults and deadlocks. Also as number of connected clients increase, the server has to deal with communications for each of the clients. Redundant communications are that not serious a problem with single client but that quickly deteriorates the performance as number of clients increase.

For Phase I we had tweaked the existing client-server controller code in ParaView, called ServerManager to support multiple clients. However, to address stability and redundant communication issues we redesigned and reimplemented the underpinnings of the ParaView for Phase II. The end result was a ServerManager implementation with more of the control logic moved to the server-side (avoiding redundancy with multiple clients). We also changed the client-server communication protocol to use Protocol Buffers [4], Google's data interchange format.

The restructuring of the ParaView ServerManager results is observable improvement on slow connections even in single client mode. We saw a performance boost of two times when creating pipeline objects such as sources, readers, filters, displays etc when compared with previous versions of ParaView.

After the ServerManager improvements, we added the collaborative features to enable collaboration between multiple clients. The highlights of this collaborative visualization infrastructure can be summarized as follows:

- Master-Slave(s) Configuration: As demonstrated in our Phase I prototype, ParaView uses a masterslave configuration when multiple clients are connected to the server. The first connected client is automatically elected master, however the leadership is transferable using the Collaboration Panel 


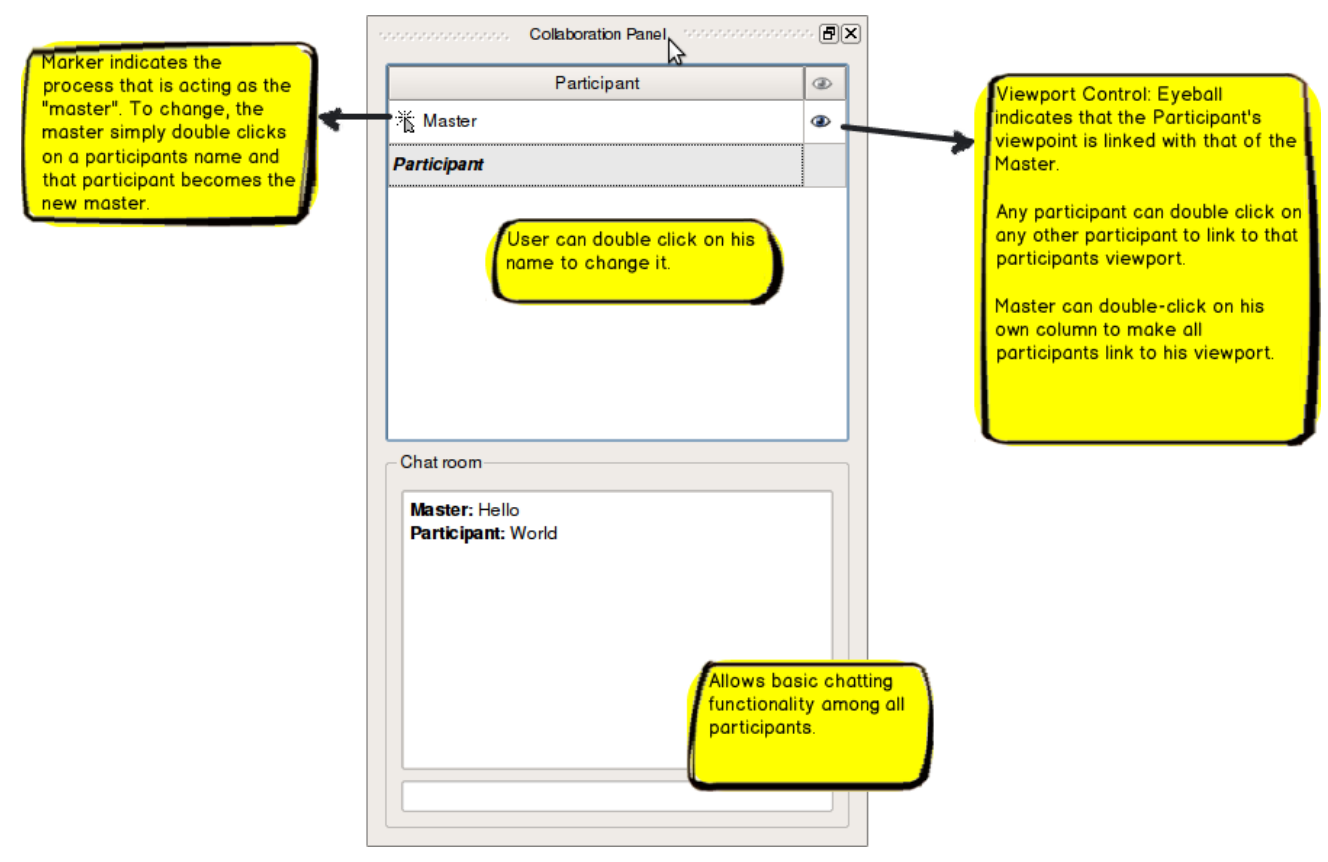

Figure 6: Collaboration Panel used to access collaboration features in ParaView.

shown in Figure 6. Collaboration Panel provides access to other collaborative features such as linking viewports and broadcasting messages to all participants.

- Heterogeneous Clients: One of the major advantages of our collaboration framework over conventional video-stream based collaboration solutions such as Adobe Connect [1] and AccessGrid [3] is that we added support for heterogeneous clients. All connected clients don't need to have the same display resolution or aspect ratios. Each client can request for rendering optimal for its screen resolution. Additional each client can decide whether it uses local rendering (delivering geometry to be rendering locally) or remote rendering (where rendering happens on server side and images are delivered to the client) separately. Thus each client can make best use of the resources available locally.

- Individual and Shared Viewpoints: Each client can render a scene based on its own view of interest. Of course, it's also possible to link a camera to any participants view so that a user can see the visualization from that participants viewport. Also, the master can make all connected participants to link their viewpoints to his own, useful for giving presentations or demonstrations. All this is accessed through the Collaboration Panel shown in Figure 6.

- Annotation: ParaView provides a mechanism to select dataset by simply clicking-and-dragging on the screen. We ensured that this mechanism works with multiple clients as well. This makes it possible for participants to highlight regions of interest for each other.

- Support for different types of views: ParaView uses different view types to show the data in different forms, for example, 3D view is used to view 3D rendering, Line Chart view is used to render XY line plots, while Spreadsheet view is used to inspect the raw data. These standard view types can be used in collaborative configurations as well. 


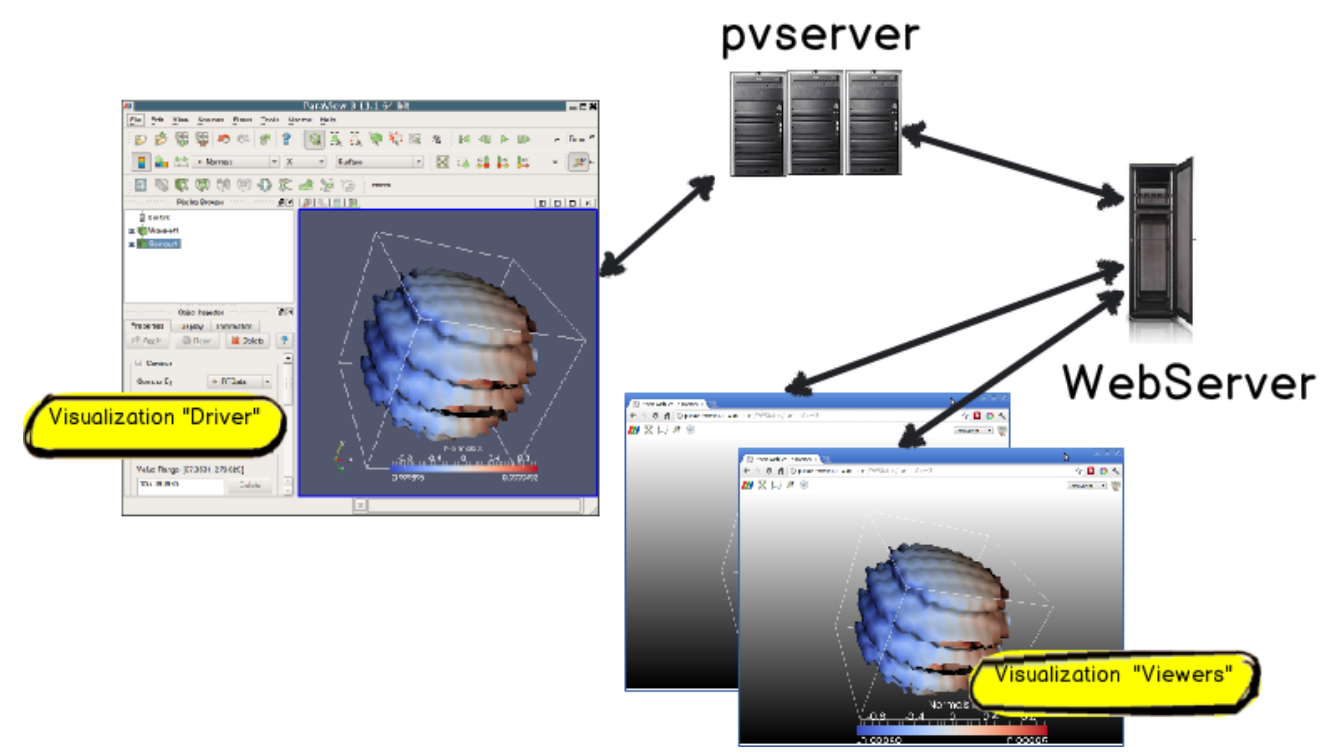

Figure 7: A demonstration of a collaborative session between a ParaView Qt client (acting as the driver) and multiple ParaViewWeb clients acting as viewers.

\subsection{Collaboration over web (e.g. with web clients)}

One of the driving use-cases for our collaboration framework was to make it easier for domain specialists who may not be familiar with visualization tools to participate in the visualization and data analysis process. With that in mind, we developed ParaViewWeb that makes it possible to create highly customized web-based applications that can be developed with the user in mind. A natural extension for that is to enable users to collaborate with each other through the web clients.

We added support for multiple ParaViewWeb clients to interact with the same visualization. Additionally, we also added support for both the ParaViewWeb client as well as the standard ParaView Qt client to the connect to the same ParaView server and share the visualization. A common use-case for this setup would be the visualization expert driving the visualization using the ParaView Qt client and all the advanced capabilities it exposes, while the domain expert or customers can be looking in on the visualization via simple web-clients as shown in Figure 7.

\subsection{Electromagnetic accelerator modeling integration}

SLAC's simulation suites, used by scientists in multiple institutions, employ a high-order finite element method to perform high-fidelity and high accuracy time-domain or frequency-domain analysis for complex accelerator structures. This method can use various basis functions, where there can be different implementations for even a single set of basis functions. SLAC uses its own implementation of a set of hierarchical Whitney basis functions. Whitney basis functions provide tangential continuity across elements, which is necessary for SLAC's codes. Because these basis functions are hierarchical (for example, orders 1 through 6 have $6,20,45,84,140,216$ coefficients, respectively), there is the option of allowing a visualization trade-off between speed using fewer coefficients vs. accuracy using more coefficients. For some analysis done at SLAC, such as post-processing and certain queries, accuracy is more important than speed, so a user adjustable tradeoff between accuracy and speed is important. The ability to visualize fields to full accuracy is needed for debugging meshes for field solving, and for seeing field discontinuities perpendicular to edges, which can arise because Whitney basis functions only guarantee continuity tangent to element edges.

ParaView supports finite elements by way of a generic VTK adaptor framework that enables specific 


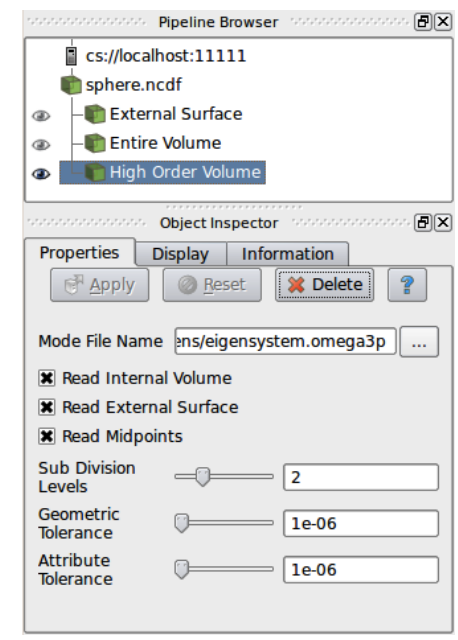

Figure 8: SLAC reader for High Order Element control UI

basis functions already implemented in other libraries to be wrapped for use in ParaView. ParaView also includes adaptive tessellation to permit the full set of ParaView's capabilities to work with higher order data [7]. This can prevent visualization artifacts which occur with linear interpolation, as shown in the figure 10 .

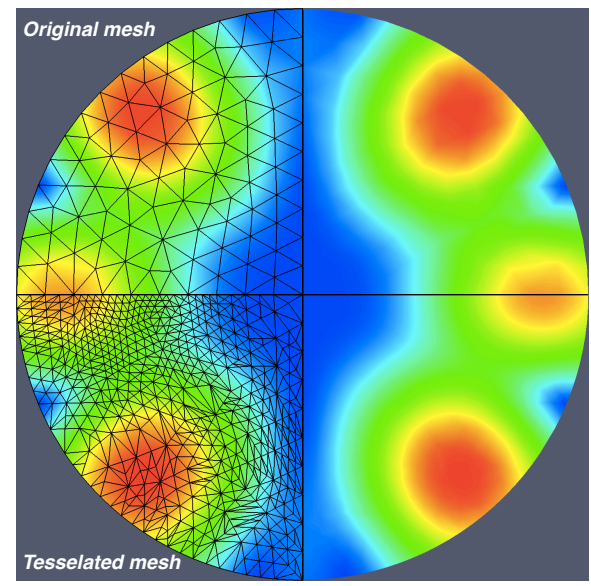

Figure 9: Dynamic tessellation based on attribute

In order to integrated the usage of the high order element used by SLAC into ParaView, we have implemented a set of VTK classes used to interconnect the mathematical implementation of the basis function into the adaptor framework of VTK. Although, to fulfill that integration a new ParaView reader plugin as been written. That new HighOrderElement reader, take two files as input; the first file represent the mesh information which remain similar to the previous SLAC file data structure; the second one is the data file that stores the whitney coefficients that are going to be used for the data field interpolation. Based on those two files, the reader generate 3 types of outputs. The first 2 outputs are regular unstructured grid on which we dynamically computed the field value for each point based on the coefficient that we load. The last output is the hight order dataset, on which a set of custom filter can be applied in order to benefit the most of the high order field integration such as stream line or contour filter. Moreover, in order to adjust the tradeoff between accuracy and speed, the current reader offer some settings used in 

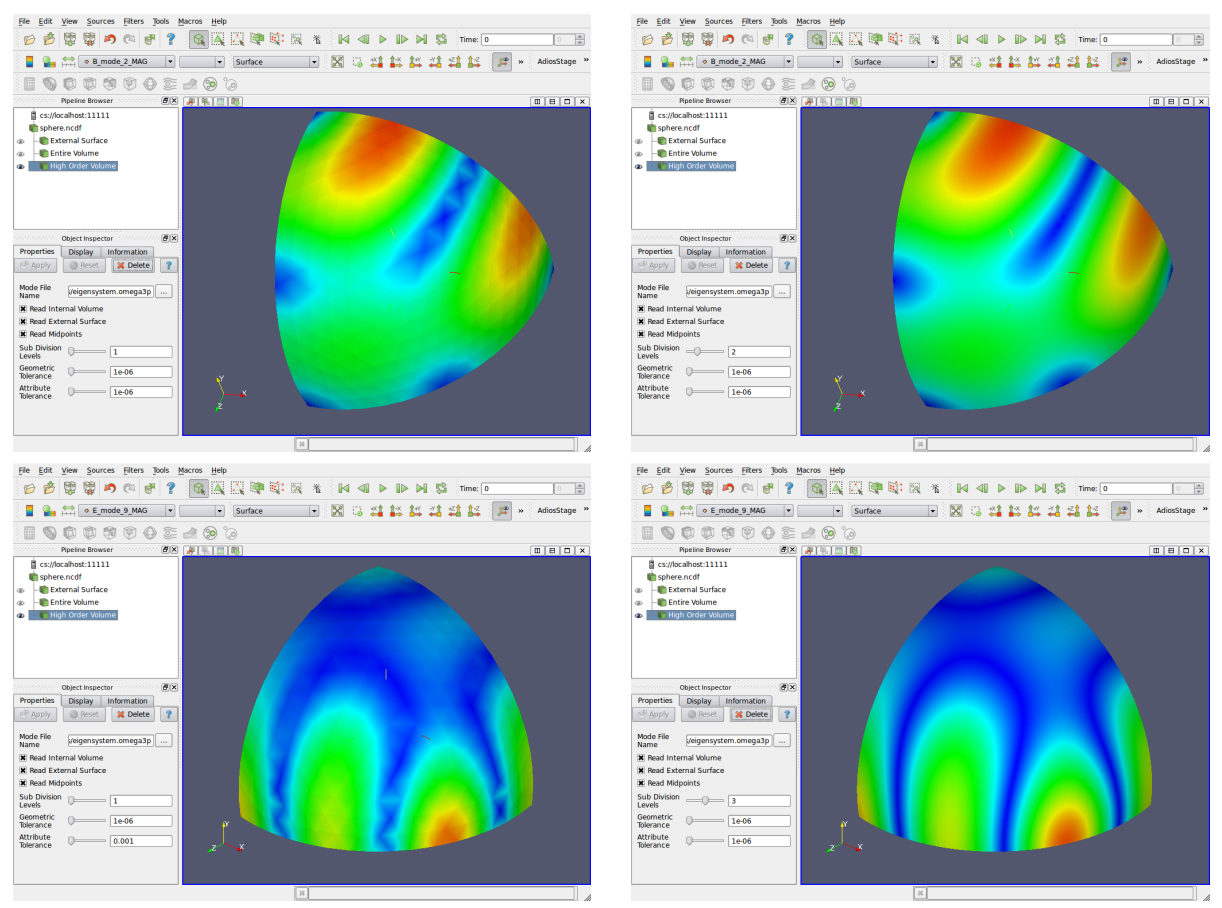

Figure 10: A selection of linear visualization compared to high order ones with automatic tessellation using ParaView to demonstrate the capabilities of the new HighOrder SLAC reader.

the tessellation process. The figure 8 show both the 3 outputs in the pipeline as well as all the parameters that can be used to adjust the expected accuracy. Depending on the accuracy setting the high order output get dynamically refined to reduce the interpolation error between the vertex of the rendered geometry.

One of the goal with that integration was to eliminate visual artifacts that could occurs due to simple linear field interpolation to benefit of a more accurate analysis. The figure 10 provide a nice highlight of that achievement, although that tessellation mechanism can consume a lot of resources both in term of memory and CPU. It's definitely a step in the right direction, but some effort are still needed to ease the adoption of such accurate environment.

As part of that effort, several improvement have been made into ParaView itself for a better support to Generic DataSet in general.

\subsection{Deployment}

We have deployed ParaViewWeb on a demo machine here at Kitware, located at Clifton Park, NY. The installation is accessible from http://paraviewweb.kitware.com. It demonstrates different sample applications developed using ParaViewWeb framework. It can also be used for demonstrating collaborative features in ParaViewWeb.

We helped several other organizations and collaborators deploy ParaViewWeb including Sandia National Laboratories, Los Alamos National Laboratories, Idaho National Laboratories. In general consensus has been that the technology is very exciting and indeed the way for the future. What needs to identified is how it integrates into the existing HPC work-flows used by researchers.

On those lines, we had started working with Texas Advanced Computing Center (TACC). TACC provides a web-based visualization platform for its users, called EnVision. The current version of EnVision uses VNC and single process visualization server. We had started assisting them with upgrading EnVision to use ParaViewWeb as the visualization engine while keeping the user interface same. However the effort had to be postponed due to funding issues on TACC's side. However, EnVision could become a 
very good demonstration platform for ParaViewWeb and we will keep working with to try to integrate the two technologies in the future.

\section{Publications}

Following is the list of publications that document components developed during this Phase II effort.

1. Remote Visualization of Large Datasets with MIDAS and ParaViewWeb at Web 3D ACM Conference, 2011 [5].

2. ParaViewWeb, A web framework for 3D Visualization and Data Processing at IADIS International Conference on Web Virtual Reality and Three-Dimensional Worlds [6].

3. ParaViewWeb - Bringing Visualization to the Web in the Kitware Source [2].

\section{Internet Sites}

We have deployed a demonstration web-site at http://paraviewweb.kitware.com that demonstrates the collaborative features of ParaView when used through the Web browser.

Detailed documentation about some of the components developed here can be found at the following links:

- http://www.paraview.org/Wiki/ParaView

- http://www.paraview.org/Wiki/ParaViewWeb

\section{References}

[1] Adobe Systems Incorporated. Adobe connect. http://www.adobe.com/products/ adobeconnect.html.

[2] U. Ayachit and S. Jourdain. Paraviewweb bringing visualization to the web. http://www.kitware.com/products/html/ ParaViewWebBringingVisualizationToTheWeb.html, July 2010.

[3] L. Childers, T. Disz, R. Olson, M. E. Papka, R. Stevens, and T. Udeshi. Access grid: Immersive group-to-group collaborative visualization, 2000.

[4] Google Inc. Protocol buffers. http://code.google.com/p/protobuf/.

[5] J. Jomier, S. Jourdain, U. Ayachit, and C. Marion. Remote visualization of large datasets with midas and paraviewweb. Web 3D ACM Conference, 2011.

[6] S. Jourdain, U. Ayachit, and B. Geveci. Paraviewweb, a web framework for 3d visualization and data processing. IADIS International Conference on Web Virtual Reality and Three-Dimensional Worlds, 2010.

[7] W. Schroeder, B. Geveci, and M. Malaterre. Compatible triangulations of spatial decompositions. Proceedings of IEEE Visualization, 102004. 